\title{
COMPORTAMIENTO DE MEZCLAS ASFÁLTICAS CERRADAS MEDIANTE LA APLICACIÓN DEL ENSAYO FÉNIX.
}

\section{BEHAVIOR DENSE ASPHALT MIXTURES BY THE FÉNIX TEST.}

\author{
Linna Marcela Neme Ardila \\ Ing. Civil, Facultad de Ingeniería, Auxiliar Investigador Grupo Geotecnia. \\ Universidad Militar Nueva Granada, \\ Bogotá, Colombia, \\ u1100861@unimilitar.edu.co \\ Óscar Javier Reyes Ortiz \\ Ing. Civil, Ph.D., Profesor Titular, Facultad de Ingeniería, Líder Grupo Geotecnia. \\ Universidad Militar Nueva Granada, \\ Bogotá, Colombia, \\ oscar.reyes@unimilitar.edu.co \\ Javier Fernando Camacho Tauta \\ Ing. Civil, Ph.D., Profesor Asociado, Facultad de Ingeniería, Investigador Grupo Geotecnia. \\ Universidad Militar Nueva Granada, \\ Bogotá, Colombia, \\ javier.camacho@unimilitar.edu.co
}

Fecha de recepción: 26 de abril de 2013

Fecha de aprobación: 27 de noviembre de 2013

\section{RESUMEN}

Las mezclas asfálticas son el material más utilizado en la fabricación de pavimentos y los ensayos que permiten caracterizarlas son costosos y demorados. Por esta razón, mediante esta investigación se planteó establecer la viabilidad del uso del ensayo Fénix en mezclas asfálticas colombianas con granulometrías del Instituto de Desarrollo Urbano (IDU) y del Instituto Nacional de Vías (INVIAS) con diferentes características. El estudio inició con la fabricación de probetas Fénix con diferentes materiales (Agregados, asfaltos, asfaltita, pavimento asfalto reciclado (RAP), cal, cemento) y su ejecución a $15{ }^{\circ} \mathrm{C}$, una velocidad de $1 \mathrm{~mm} / \mathrm{min}$ y la medición de los parámetros del ensayo. De los resultados obtenidos de resistencia a tracción, índice de rigidez a tracción e índice de energía, área elástica y área 
de fluencia, se estableció que el ensayo Fénix es un procedimiento eficaz, eficiente, económico, rápido y sencillo para determinar las propiedades mecánicas y dinámicas de las mezclas asfálticas estudiadas, especialmente mediante el análisis de los parámetros de las curvas carga-desplazamiento, irrelevantemente de la mezcla asfáltica fabricada.

Palabras clave: Asfaltitas, Ensayo Fenix, Índice de Rigidez a Tracción, Pavimento Asfalto Reciclado, Resistencia a Tracción.

\section{ABSTRACT}

Asphalt mixtures are the most commonly used material in roads. Usually, mechanic tests to characterize them are expensive and time consuming. For this reason, it was studied the feasibility of using the Fenix test to evaluate Colombian asphalt mixtures elaborated under specifications of the Urban Development Institute of Bogotá (IDU) and the Colombian National Roads Institute (INVIAS). The study began with the manufacture of specimens with different materials (aggregates, bitumen, asphaltite, reclaimed asphalt pavement (RAP), lime and cement). Fenix test were carried out at $15^{\circ} \mathrm{C}$ and velocity of $1 \mathrm{~mm} / \mathrm{min}$. From the results of tensile strength, tensile stiffness index and energy index, elastic area and flow area, it was established that the Fenix is an effective, efficient, economical, fast and easy test to measure the mechanical and dynamical properties of the asphalt mixtures included in the research. Moreover, analyses of the load-displacement curves were very useful for all asphalt mixtures.

Key words: Asphaltites, Fenix test, tensile stiffness index, recycled asphalt pavement, tensile strength.

\section{INTRODUCCIÓN}

La infraestructura vial es una componente importante en el desarrollo económico y social de un territorio, pues permite la comunicación e interrelación entre centros poblados, así como el intercambio de bienes y servicios. En este orden de ideas, la estructura de pavimento, como parte de la infraestructura vial juega un papel predominante, ya que su objetivo es ofrecer a los usuarios un desplazamiento cómodo, seguro y económico, por lo que se puede definir que las vías son la columna vertebral del transporte y la comunicación, y su construcción se vuelve estratégica para un país o región [1-2].

Las estructuras de pavimento flexible para calles, carreteras y autopistas están sometidas a cargas repetitivas impuestas por los vehículos y las condiciones climatológicas, 
las cuales producen deterioro en las propiedades de las capas y por ende son objeto de estudio. Este tipo de cargas pueden ser estáticas o dinámicas, de corta o larga duración, que llevan a deteriorar la estructura del pavimento en un periodo de tiempo [3-4].

Por las circunstancias de deterioro de los pavimentos, se han desarrollado múltiples ensayos de laboratorio, tales como ensayo a tracción indirecta (RTI), módulo resiliente (MR), ahuellamiento, fatiga, resistencia conservada, resistencia a la deformación permanente, entre otros. Sin embargo, estos ensayos son costosos, necesitan de un tiempo elevado de ejecución y en oportunidades solo brindan un tipo de comportamiento de las mezclas asfálticas. Normas UNE-EN [5]. Es por esto, que la Universidad Politécnica de Cataluña (UPC) desarrolló y puso a punto un nuevo ensayo denominado Fénix, el cual fue creado en el Proyecto de Investigación Estratégica de carreteras más seguras y sostenibles, Acrónimo: FÉNIX. Valdés et al [6]. Este ensayo permite estudiar las mezclas asfálticas densas, semidensas, gruesas, entre otras, de una manera fácil, rápida, sencilla y económica de fabricar y ejecutar [7].

En Colombia son escasas las investigaciones que se han realizado sobre el fenómeno de deterioro en las mezclas asfálticas, debido ante todo, a la falta de equipos apropiados para llevar a cabo mediciones en campo y en el laboratorio y al escaso presupuesto destinado para estas investigaciones [2].
Es por esto, que con base en las investigaciones desarrolladas con el ensayo Fénix, se buscó establecer la viabilidad de su uso y permitir la caracterización de las propiedades mecánicas y dinámicas de mezclas asfálticas con granulometrías cerradas colombianas del Instituto Nacional de Vías (INVIAS) [8] y granulometrías del Instituto de Desarrollo Urbano (IDU) [9], fabricadas con agregados y asfaltos convencionales, asfaltitas, RAP, reemplazo de filler por cemento o cal entre otras. Adicionalmente, se pretende de una manera simple, sencilla y económica, buscar implementar un ensayo que permita evaluar las mezclas asfálticas sin la necesidad de tener múltiples equipos de laboratorio y el empleo de recursos y tiempos elevados.

\section{METOdOLOGÍA}

La investigación se desarrolló siguiendo la metodología que se presenta en la Figura 1 , iniciando con la caracterización de los materiales (Asfaltos, agregados, asfaltitas, RAP, cemento, cal). Posteriormente, se fabricaron probetas con granulometrías densas del IDU e INVIAS, con asfaltitas, reemplazo de filler por cemento o cal, RAP y compactadas a diferentes niveles de energía. A continuación, se cortaron las probetas para poder realizar el ensayo Fénix y establecer los parámetros del ensayo. Finalmente, se calcularon y analizaron los parámetros, con el objeto de establecer el comportamiento mecánico de las mezclas asfálticas estudiadas y establecer la viabilidad de su aplicación. 


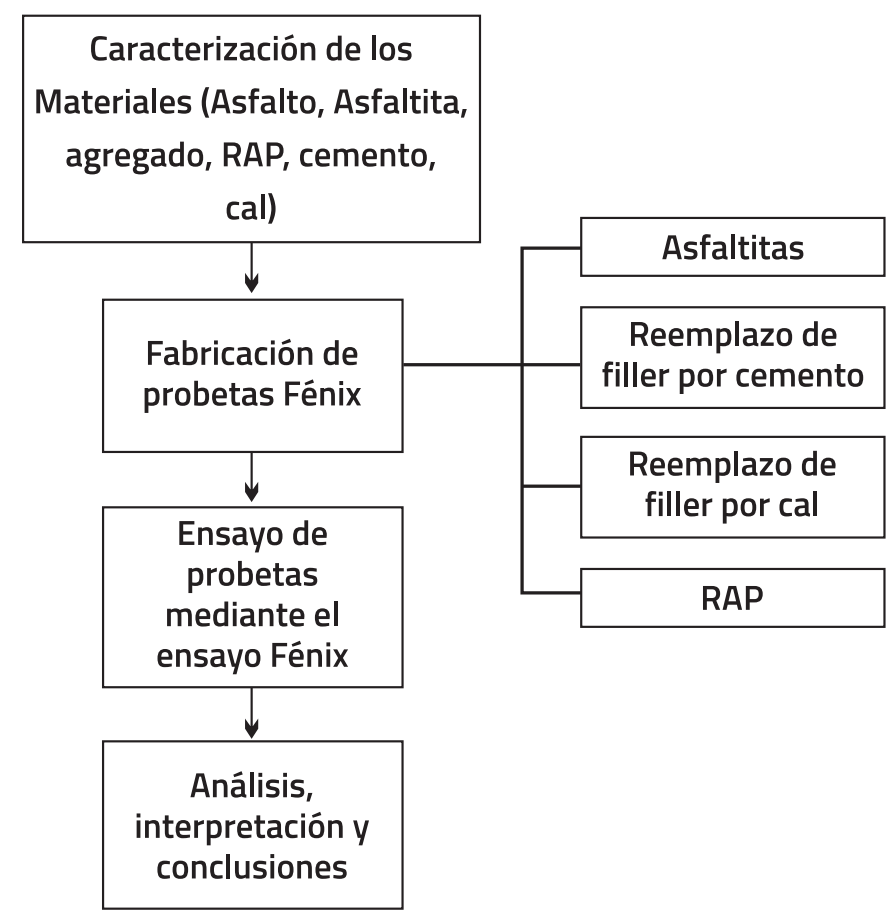

Figura 1. Diagrama de la metodología de la investigación

Fuente: Original de los autores

\subsection{CARACTERIZACIÓN DE LOS MATERIALES USADOS EN EL ESTUDIO}

Para la fabricación de las probetas de mezclas asfálticas del estudio, se usaron granulares provenientes de la cantera del Río
Tunjuelito (Tabla 1). [8-10], asfaltitas de las minas de Boyacá (Tabla 2). Castillo [11], asfaltos de diferente penetración (Tabla 3) [10-13] y reemplazos de filler por cemento y cal (Tabla 4) [10-14].

Tabla 1. Características de los agregados utilizados.

\begin{tabular}{|l|c|c|}
\hline \multicolumn{1}{|c|}{ ENSAYO } & NORMA & RESULTADO TUNJUELITO \\
\hline Equivalente de arena [\%] & INV. E-133 & 57,00 \\
\hline Resistencia al desgaste [\%] & INV. E-218 & 29,90 \\
\hline Peso específico aparente agregado fino [\%] & INV. E-222 & 2,60 \\
\hline Absorción agregado fino [\%] & INV. E-222 & 1,10 \\
\hline Peso específico aparente agregado grueso [\%] & INV. E-223 & 2,59 \\
\hline Absorción agregado grueso [\%] & INV. E-133 & 1,60 \\
\hline
\end{tabular}

Fuente: INVIAS, 2002. Rodríguez, 2011. [8,10] 
Tabla 2. Características de las asfaltitas.

\begin{tabular}{|c|c|c|c|c|c|}
\hline \multirow{2}{*}{ MUESTRA } & $\begin{array}{c}\text { PESO } \\
\text { INICIAL } \\
\mathbf{( g )}\end{array}$ & $\begin{array}{c}\text { PESO } \\
\text { CENTRIFUGADO } \\
\mathbf{( g )}\end{array}$ & $\begin{array}{c}\text { DIFERENCIA } \\
\mathbf{( g )}\end{array}$ & \% ASFALTO & PROMEDIO (\%) \\
\hline 1 & 1500,17 & 1392,38 & 107,79 & 7,74 & \multirow{2}{*}{7.5} \\
\hline 2 & 1500,34 & 1396,00 & 104,34 & 7,47 & \\
\hline 3 & 1500,09 & 1399,96 & 100,13 & 7,15 & \\
\hline
\end{tabular}

Fuente: Castillo, Martínez, 2011. [11]

Tabla 3. Características de los asfaltos.

\begin{tabular}{|l|c|c|c|}
\hline \multicolumn{1}{|c|}{ ENSAYO } & NORMA & ASFALTO 1 (60/70) & ASFALTO 2 (80/100) \\
\hline Penetración (1/10 mm) & INV. E-706 & 63 & 89 \\
\hline Ductilidad (cm) & INV. E-702 & 120 & 133 \\
\hline Viscosidad (poises) & INV. E-716 & 1500 & 1250 \\
\hline Punto de ablandamiento ( $\left.{ }^{\circ} \mathrm{C}\right)$ & INV. E-712 & $47^{\circ} \mathrm{C}$ & $52^{\circ} \mathrm{C}$ \\
\hline Punto de llama e ignición $\left({ }^{\circ} \mathrm{C}\right)$ & INV. E-709 & $235^{\circ} \mathrm{C}$ y $245^{\circ} \mathrm{C}$ & $220^{\circ} \mathrm{C}$ y $225^{\circ} \mathrm{C}$ \\
\hline
\end{tabular}

Fuente: INVIAS, 2002. Ostos, Duarte, Reyes, 2011, Bastidas, 2011. [11-15-16]

Tabla 4. Características del cemento y la cal.

\begin{tabular}{|l|c|c|}
\hline \multicolumn{1}{|c|}{ ENSAYO } & NORMA & RESULTADO \\
\hline Peso específico (Cemento) & INV. E-307 & $3000 \mathrm{~kg} / \mathrm{m3}$ \\
\hline Densidad aparente (Cal) & INV. E-225 & $2980 \mathrm{~kg} / \mathrm{m3}$ \\
\hline
\end{tabular}

Fuente: INVIAS, 2002, Suárez, 2012 [17]

\subsection{Fabricación de las probetas.}

Para el desarrollo del estudio, se fabricaron muestras de mezclas asfálticas con granulometrías cerradas md-10, md-12, md20 del IDU y mdc-2 del INVIAS, utilizando asfalto de diferente penetración 60/70 y 80/100, agregados vírgenes y de recuperación (RAP), asfaltitas de las minas de Boyacá, reemplazo de filler por cemento y cal en diferentes porcentajes $(100 \%, 75 \%, 50 \%$ y $0 \%$ ) y muestras compactadas con diferentes niveles de energía en el compactador giratorio (100, 200 y 300 giros). Todas estas probetas, fueron divididas por la mitad y se les realizó la fisura de $5 \mathrm{~mm}$ en la mitad, para ser pegadas a las placas de acero con el epóxico y posteriormente, ser ensayadas a una velocidad de $1 \mathrm{~mm} / \mathrm{min}$ y a una temperatura de $15^{\circ} \mathrm{C}$. 


\subsection{Ensayo Fénix}

El procedimiento del ensayo Fénix consiste en fabricar una probeta cilíndrica y dividirla en su mitad para posteriormente, realizar una fisura de $5 \mathrm{~mm}$ de longitud en la parte media de la probeta (Figura 2). Acto seguido, la probeta se pega con un epóxico a las placas de acero (Figura 3). La separación existente entre las placas de acero debe ser aproximadamente de $5 \mathrm{~mm}$. Una vez la probeta esté fabricada y puesta en el dispositivo (Figura 4), se procede a ejecutar el ensayo que consiste en tirar de las placas en sentido vertical a una velocidad constante de $1 \mathrm{~mm} / \mathrm{min}$ y medir la carga durante la ejecución del ensayo. [15-17]
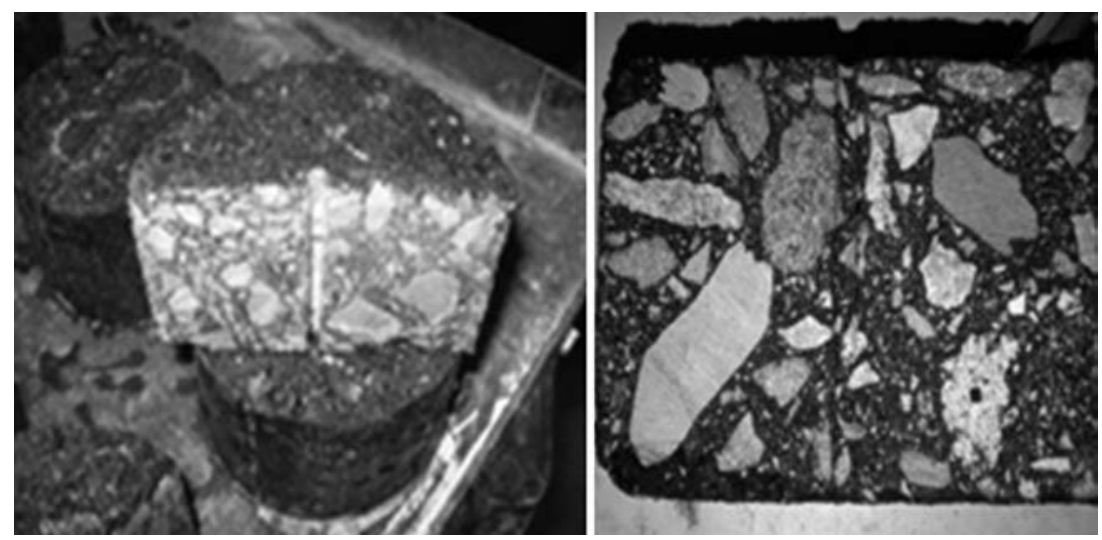

Figura 2. Inducción de fisura de $5 \mathrm{~mm}$ en la parte media

Fuente: Original de los autores
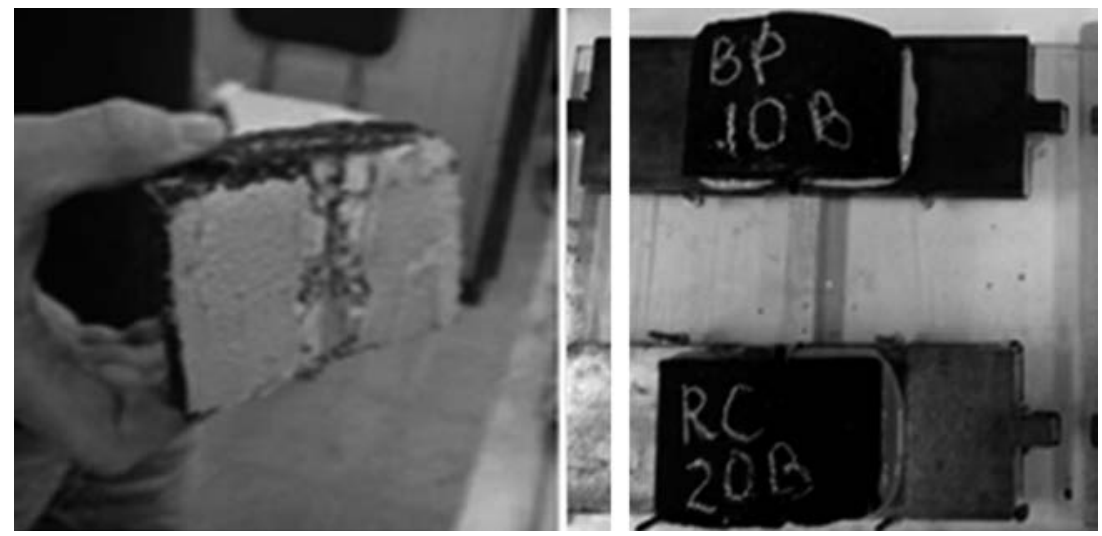

Figura 3. Unión probeta con placas de acero.

Fuente: Original de los autores 


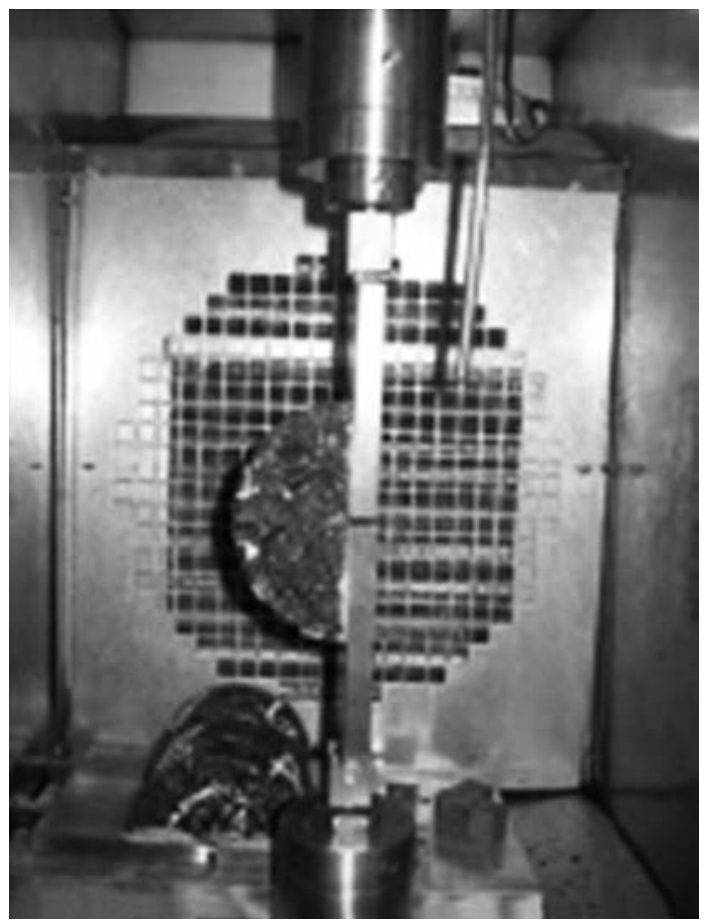

Figura 4. Disposito para la ejecución del ensayo Fénix. [16]

Fuente: Valdés, 2009.

Los parámetros que se obtienen de la curva observan en la Figura 5 y están descritos a carga-desplazamiento del ensayo Fénix, se continuación: [16,17]
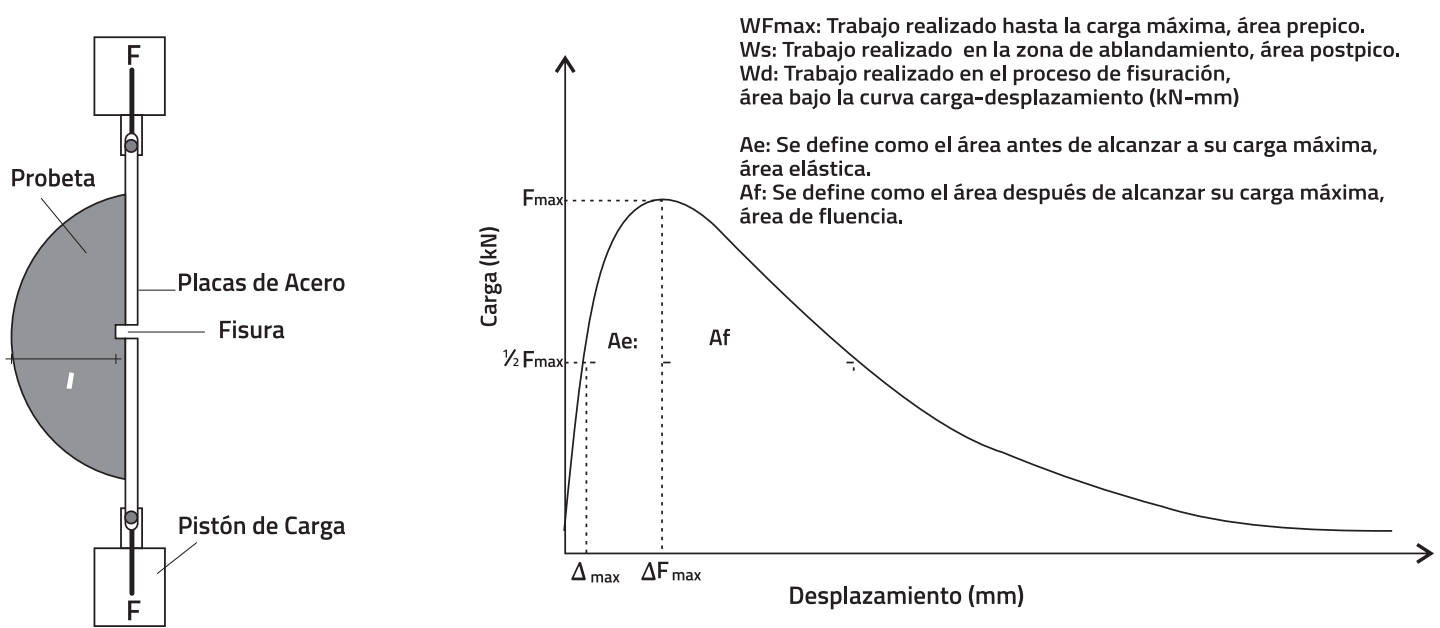

Figura 5. Curva carga-desplazamiento del ensayo Fénix y parámetros del ensayo. [16, 17]

Fuente: Valdés, 2009, Reyes, 2010. 
Carga máxima a tracción (Fmax, kN): Se define como la carga máxima registrada en el ensayo a tracción directa.

\section{Desplazamiento a carga máxima ( $\Delta \mathrm{Fmax}$,} $\mathbf{m m})$ : Se define como el desplazamiento entre el inicio de la carga y la carga máxima a tracción.

Resistencia a tracción (RT, MPa): Se define como la relación entre la carga máxima registrada, (Fmax), y el área que resiste las cargas. La resistencia a tracción se calcula utilizando la Ecuación. (1).

$$
R T=1000 *\left(\frac{F \max }{l * h}\right)
$$

Dónde:

RT: Resistencia a tracción (MPa)

Fmax: Carga máxima registrada (kN)

h: Espesor de la probeta (mm)

\section{I: Ancho de la probeta (mm)}

Índice de rigidez a tracción (IRT, kN/mm): Se define como la relación entre la mitad de la carga máxima, $1 / 2$ Fmax, y el desplazamiento a la carga máxima, $\Delta \mathrm{m}$. Este índice indica una pseudo-rigidez del material, mostrando qué tan flexible o rígida es la mezcla asfáltica evaluada. A mayor índice de rigidez a tracción, menos flexible es la mezcla. El índice de rigidez de tracción se calcula de acuerdo a la Ecuación. (2).

$$
\mathrm{IRT}=\frac{\frac{1}{2} * \mathrm{~F} \max }{\Delta \mathrm{m}}
$$

Dónde:

IRT: Índice de rigidez a tracción (kN/mm)

Fmax: Carga máxima registrada (kN)

$\Delta \mathrm{m}$ : Desplazamiento antes a la carga máxima a $\frac{1}{2}$ Fmax (mm)

Índice de energía (IE, J/m²): Se define como el trabajo realizado en la zona de ablandamiento dividido por el área de la probeta, todo esto multiplicado por el trabajo realizado en la zona de ablandamiento y el trabajo realizado en el proceso de fisuración, área bajo la curva carga-desplazamiento. Este índice tiene la finalidad de evaluar la cantidad de energía disipada por el material, una vez que éste ha alcanzado su resistencia máxima, ponderando las mezclas que sean capaces de disipar mayor energía en su fase de ablandamiento, respecto de la disipada en su fase de carga inicial hasta la carga máxima. A mayor índice de energía, más energía dúctil tiene la mezcla. Su expresión de cálculo se observa en la Ecuación. (3). 


$$
\mathrm{IE}=\frac{\mathrm{Ws}}{\mathrm{h} * \mathrm{I}} *\left(\frac{\mathrm{Ws}}{\mathrm{Wd}}\right)
$$

Dónde:

IE: Índice de energía $\left(\mathrm{J} / \mathrm{m}^{2}\right)$

Ws: Trabajo realizado en la zona de ablandamiento, postpico (kN-mm).

Wd: Trabajo realizado en el proceso de fisuración, área bajo la curva carga-desplazamiento (kN-mm)

h: Espesor de la probeta (m)

I: Ancho de la probeta (m)

Área elástica (kN-mm): Se define como el área antes de alcanzar a su carga máxima.

Área de fluencia (kN-mm): Se define como el área después de alcanzar su carga máxima.

\section{ANÁLISIS DE DATOS}

A partir de los resultados del ensayo Fénix y de establecer los parámetros de las curvas carga-desplazamiento de este ensayo, para las diferentes muestras utilizadas en la investigación, se pudieron obtener los siguientes resultados:
En la Figura 6, se observan los resultados de las curvas carga-desplazamiento de las muestras fabricadas con reemplazo de filler por cemento en diferentes porcentajes $(0,75,100 \%)$ y con asfalto de penetración 60/70. Del análisis de las curvas carga-desplazamiento y los parámetros carga máxima a tracción (Fmax) e índice de energía (IE), se muestra claramente que a medida que existe un aumento en el porcentaje de reemplazo de filler mineral por cemento, aumenta la resistencia y disminuye la ductilidad. De igual manera, se muestra que la probeta con $100 \%$ de reemplazo, tiene una carga máxima de $0,667 \mathrm{kN}$, valor en un $80 \%$ mayor que la muestra con $75 \%$ de reemplazo $(0,553$ $\mathrm{kN})$. Adicionalmente, la probeta con $0 \%$ de reemplazo evidencia la menor resistencia con una carga máxima de 0,328 kN. Así mismo, el índice de energía (IE) aumenta en las mezclas más dúctiles como en el caso de la probeta con $0 \%$ de reemplazo $\left(0,778 \mathrm{~J} / \mathrm{m}^{2}\right)$, que comparado a la de $100 \%$ de reemplazo, ésta disminuye en un 30\% $\left(0,461 \mathrm{~J} / \mathrm{m}^{2}\right)$. Estos resultados manifiestan que los parámetros del ensayo Fénix indican el comportamiento de mezclas asfálticas al reemplazar relleno mineral por cemento, haciendo sensible el ensayo con estos cambios y por ende, la viabilidad de su uso para establecer cambios en sus propiedades. 


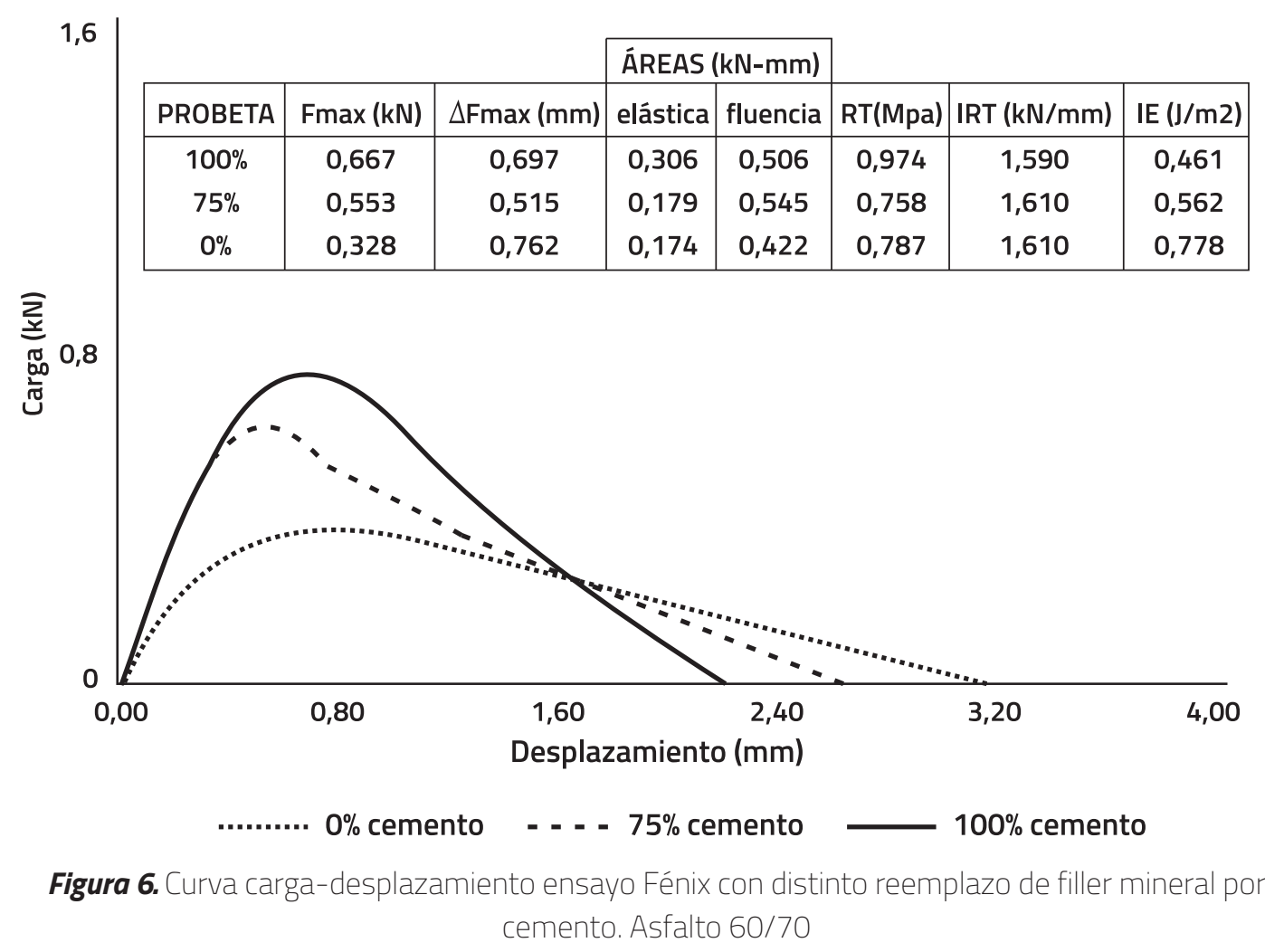

Fuente: Original de los autores

En la Figura 7, se muestran las probetas con las mismas condiciones enunciadas en la Figura 6 (Reemplazo de filler mineral por cemento a $0,75,100 \%$ ), pero fabricado con asfalto de penetración 80/100. Del análisis de la resistencia máxima y desplazamiento a resistencia máxima de las curvas de las Figuras 6 y 7 , se puede observar que la ductilidad es mayor en las probetas con asfalto 80/100 y que la resistencia máxima es superior en las probetas con asfalto $60 / 70$ y el reemplazo del $100 \%$. Por lo anterior, se evidencia la sensibilidad del ensayo al cambiar el tipo de asfalto. De otra parte, el índice de rigidez a tracción (IRT), indica qué tan flexible es la muestra y por lo tanto a mayor índice de rigidez a tracción, menos flexible es la muestra. En el caso de la probeta con $100 \%$ de reemplazo, tiene mayor valor $(1,334 \mathrm{kN} / \mathrm{mm})$ que comparado con la probeta del $75 \%$ de reemplazo $(1,290 \mathrm{kN} / \mathrm{mm})$, se observó una reducción de un $50 \%$ del índice de rigidez a tracción con la muestra a $0 \%$ de reemplazo, se establece que el índice decrece un $60 \%$ con respecto al 100\%. Estos resultados manifiestan que los parámetros del ensayo pueden evidenciar cambios en la flexibilidad de una mezcla asfáltica, al reemplazo filler por cemento. 


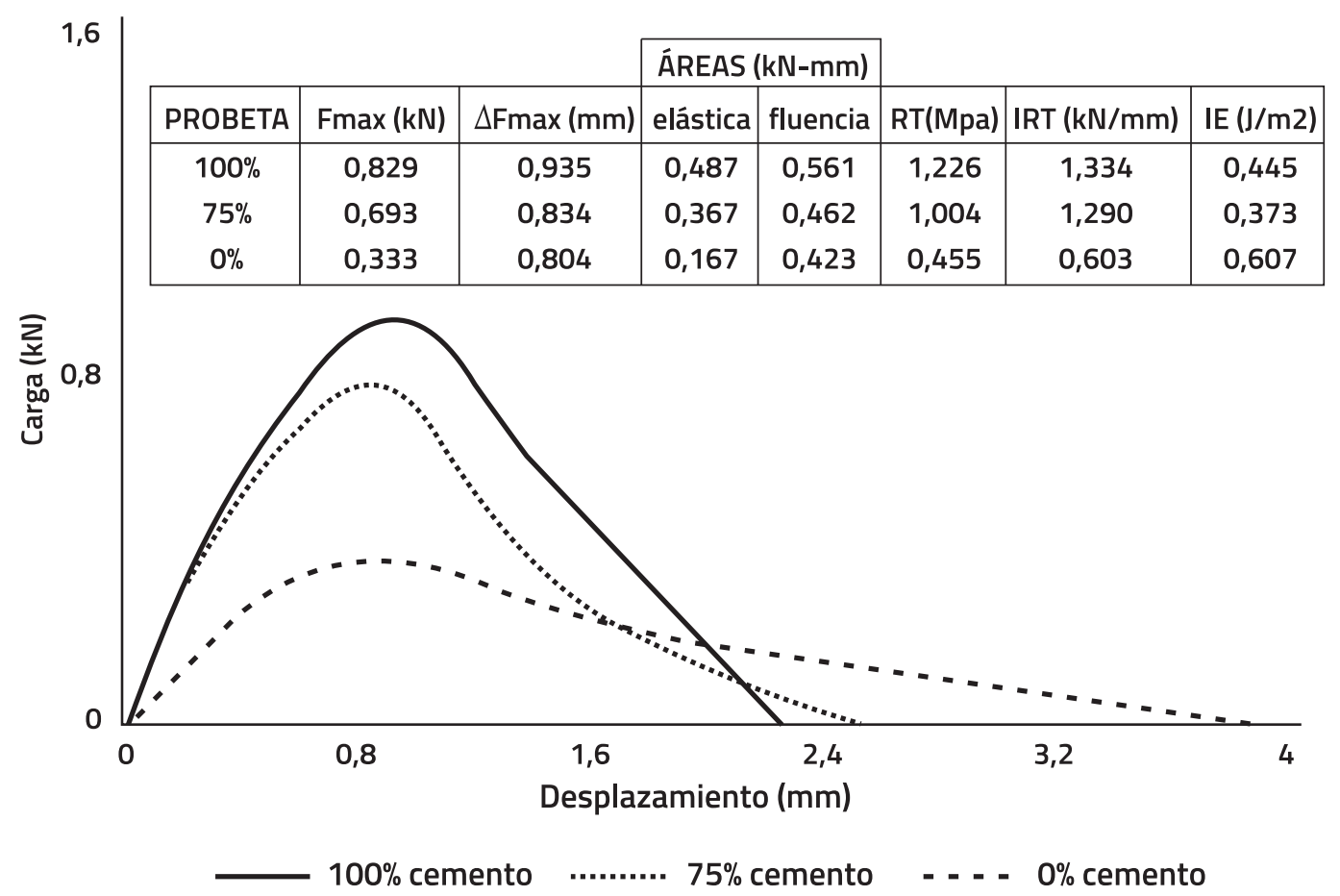

Figura 7. Curva carga-desplazamiento ensayo Fénix con distinto reemplazo de filler mineral por cemento. Asfalto 80/100

Fuente: Original de los autores

En la Figura 8, se observan las curvas carga-desplazamiento de las probetas fabricadas con diferentes niveles de compactación (100, 200 y 300 giros). Del análisis de los resultados se puede establecer que a mayor cantidad de giros, se obtiene mayor carga máxima a tracción (Fmax) y menor desplazamiento ( $\Delta$ Fmax). Adicionalmente, el ensayo muestra que la resistencia a la tracción directa (RT) aumenta con el nivel de compactación, siendo de 0,665 MPa a 100 giros, 0,976 MPa 200 giros y 1,232 MPa a 300 giros. Así mismo, se observa que el área de fluencia es mayor en las muestras más dúctiles, como se muestra en la probeta compactada a 100 giros, con un valor de 0,663 kN-mm, comparado a 0,553 kN-mm con 300 giros. Con los resultados obtenidos, se puede establecer que el ensayo Fénix ante los cambios en los niveles de compactación, es capaz de diferenciar el comportamiento de las mezclas asfálticas. 


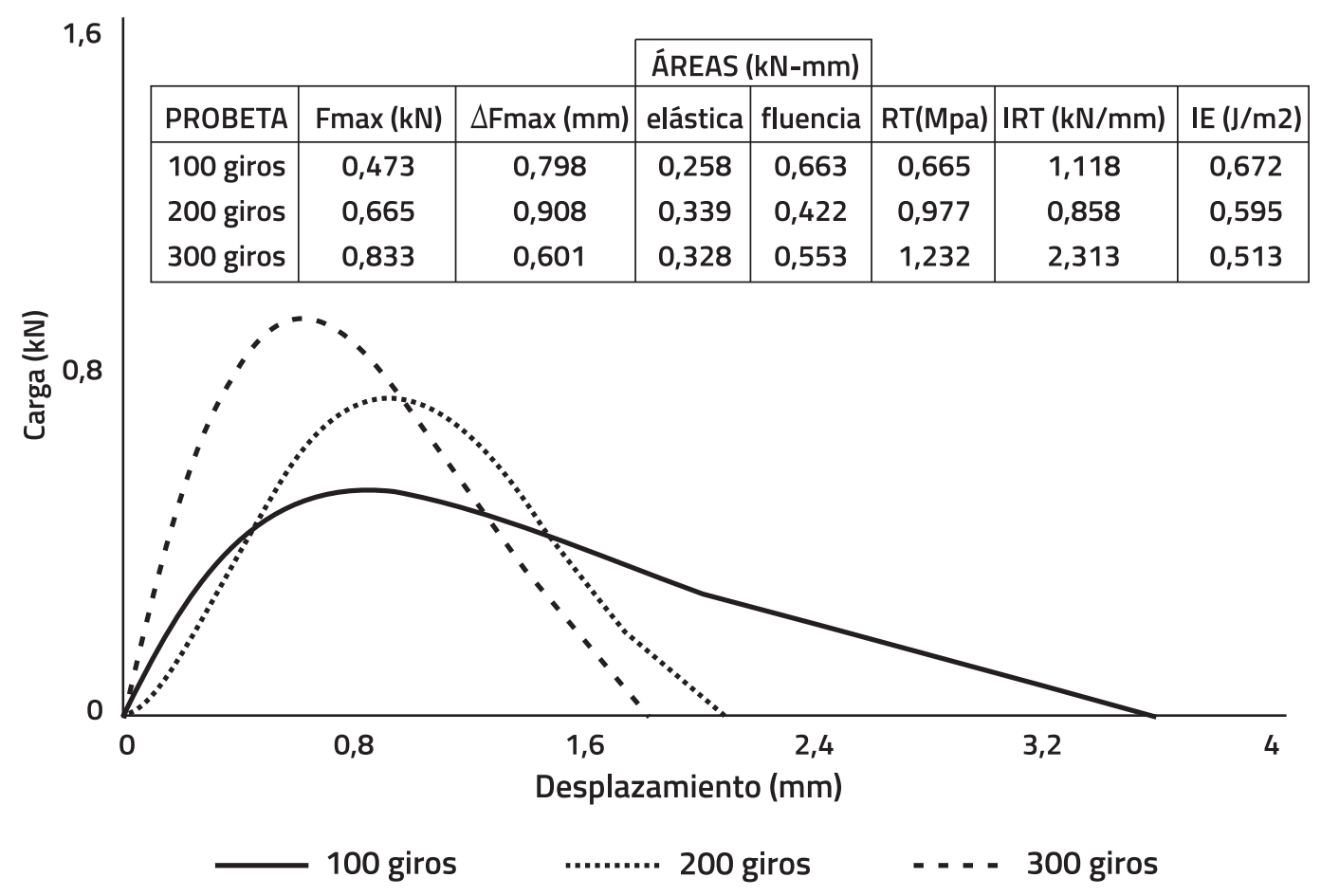

Figura 8. Curva carga-desplazamiento ensayo Fénix con diferentes niveles de compactación. Asfalto 60/70

Fuente: Original de los autores

En la Figura 9, se evidencia la respuesta del comportamiento mecánico de mezclas asfálticas cerradas fabricadas con diferentes materiales (Asfaltitas, RAP y asfalto 60/70, y agregado natural), cuyas características mecánicas son totalmente diferentes, debido al origen de sus materiales. En los resultados de las curvas carga-desplazamiento, se muestra que la asfaltita presenta la menor resistencia, mientras que el RAP la mayor. Se establece claramente que el ensayo distingue las muestras con gran rigidez y ruptura súbi- ta (RAP), como los ensayos de muestras de baja resistencia y muy dúctiles (Tipo Asfaltitas). Finalmente, en la parte media se encuentra la curva de la muestra fabricada con asfalto 60/70 y agregado natural, la cual presenta una carga y una deformación media. Estos resultados ponen de manifiesto que el ensayo Fénix y sus parámetros evidencian los cambios de rigidez y tipo de fallo (Dúctil o frágil) de las mezclas asfálticas y por ende, son un indicador de su comportamiento. 


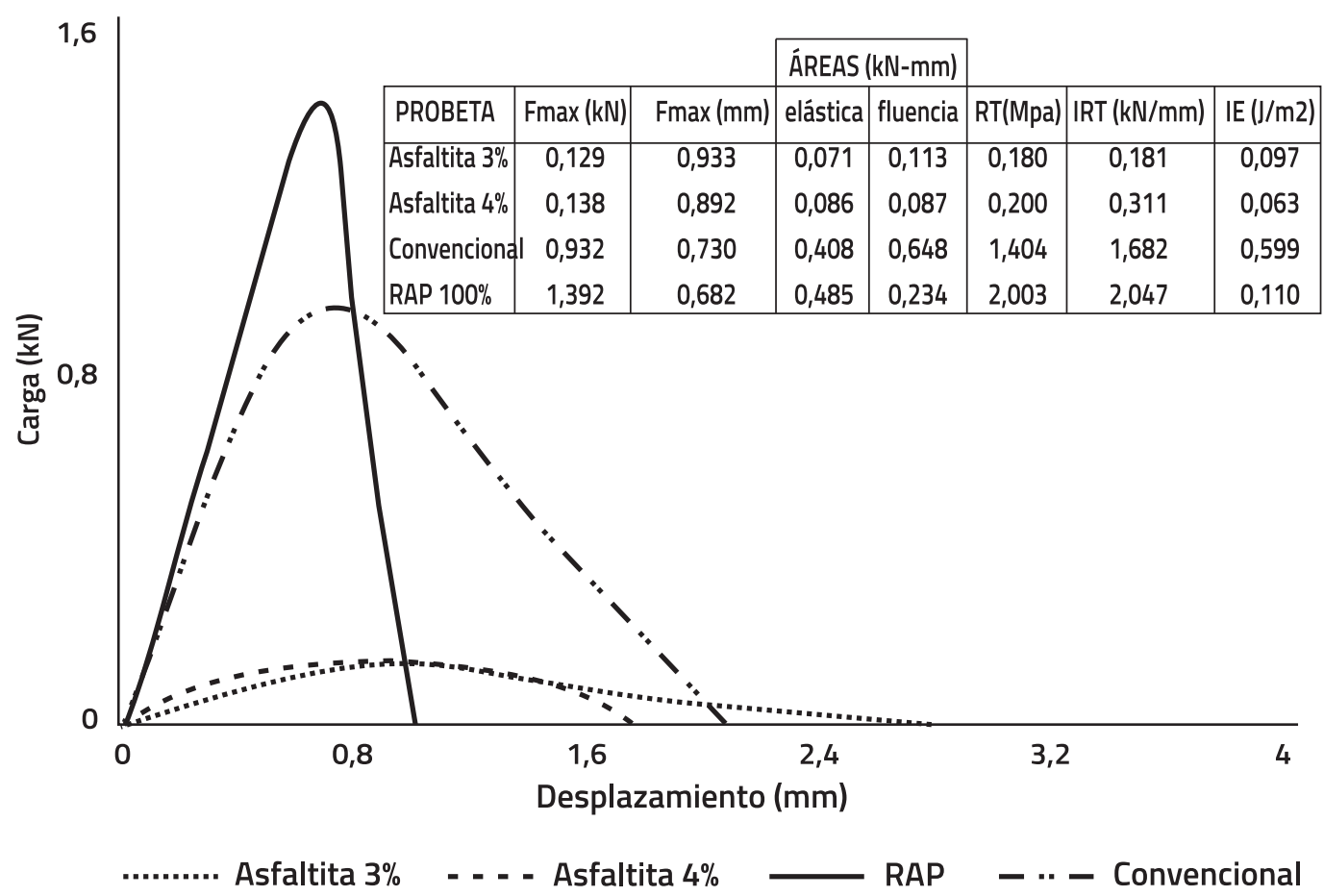

Figura 9. Curva carga-desplazamiento ensayo Fénix con diferentes materiales (Asfaltita, RAP. Asfalto 60/70 y agregado natural).

Fuente: Original de los autores

En la Figura 10, se pueden ver los resultados de las probetas fabricadas con reemplazo de filler mineral por cal en diferentes porcentajes $(100 \%, 75 \%$ y $50 \%$ ). De las curvas carga-desplazamiento, se muestra que a medida que aumenta el porcentaje de reemplazo por cal, la resistencia aumenta y la deformación disminuye, igual comportamiento observado en las muestras con reemplazo de cemento (Figura 6). Además, se establece que el índice de rigidez a tracción (IRT) presenta mayor valor en la probeta con $100 \%$ de reemplazo $(1,610 \mathrm{kN} / \mathrm{mm})$ y decrece proporcionalmente con los demás valores de reemplazo, siendo el menor a $0 \%(0,460$ $\mathrm{kN} / \mathrm{mm})$. La respuesta de los parámetros del ensayo Fénix muestra evidentemente que es viable caracterizar las mezclas asfálticas con reemplazos en diferentes porcentajes y materiales, evidenciando la sensibilidad del mismo para caracterizar las mezclas asfálticas. 


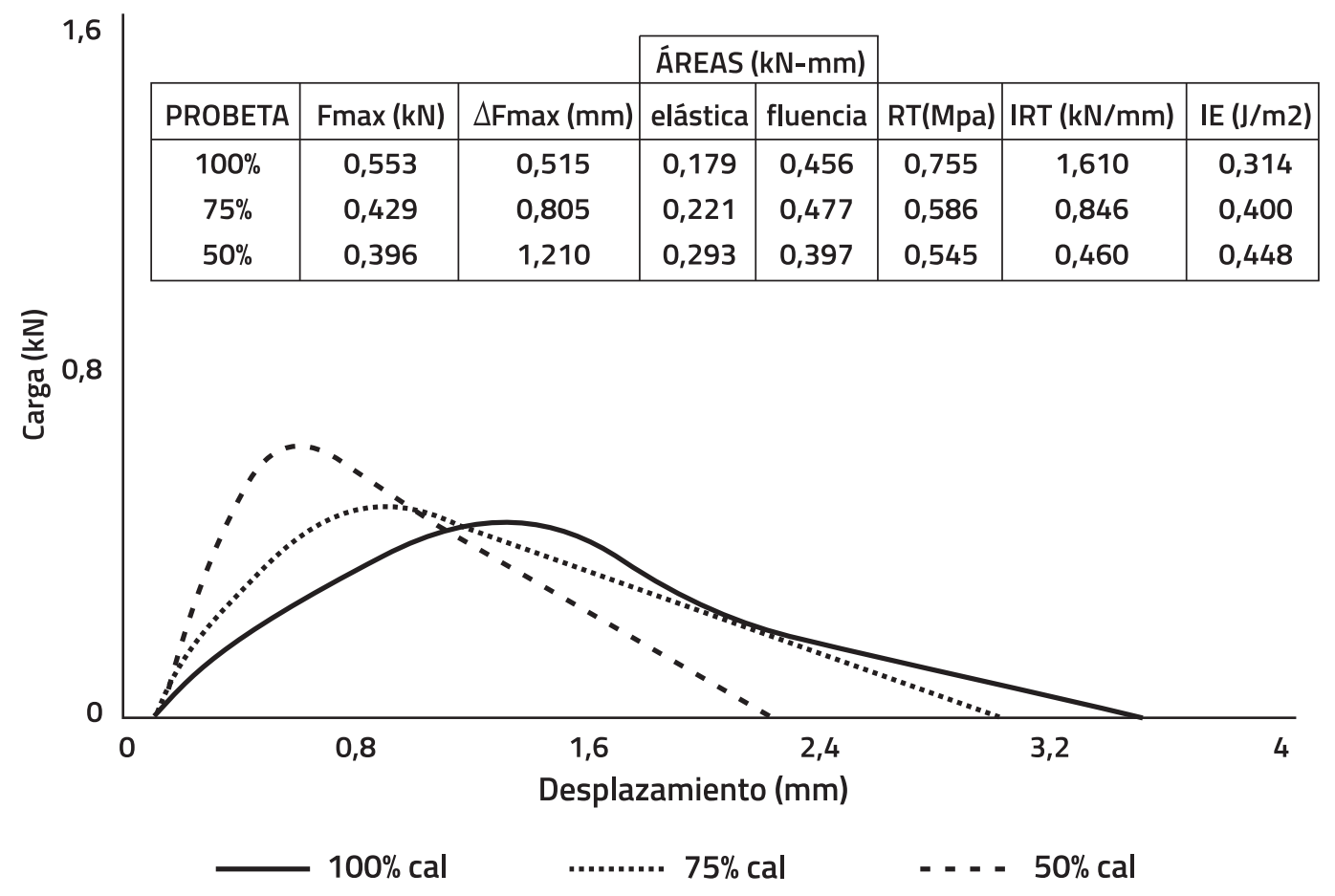

Figura 10. Curva carga-desplazamiento ensayo Fénix con distinto reemplazo de filler mineral por cal. Asfalto 60/70.

Fuente: Original de los autores

En la Figura 11, se grafican los parámetros de energía elástica y de fluencia de las probetas fabricadas con distintos materiales (Asfaltitas, RAP, asfalto 60/70, agregado natural, reemplazo de filler mineral por cemento o cal en diferentes porcentajes y con diferentes niveles de compactación). Estos parámetros muestran el comportamiento elástico y de fluencia de las muestras ensayadas y de su análisis, se observa que la probeta fabricada con RAP tiene el área elástica mayor (0,485 kN-mm), mostrando su gran rigidez. Sin embargo, su área de fluencia es baja dado su comportamiento frágil $(0,234$ kN-mm). En el caso de las muestras con asfaltitas, las áreas son similares y pequeñas, manifestando que su nivel de disipación de energía es baja, similar en la zona rígida y de ablandamiento.

En el caso de las muestras fabricadas con reemplazo de filler mineral por cemento o cal a diferentes porcentajes, se observa que las probetas presentan mayor disipación de energía en la zona de fluencia, irrelevantemente el reemplazo y el material. Así mismo, se evidencia que para el porcentaje alto de reemplazo de cemento $(100 \%)$, la energía elástica es mayor y en la muestra patrón (0\%) es menor. De otra, 
para las probetas fabricadas con diferentes niveles de energía, se observa un aumento proporcional entre el número de giros de compactación y la energía elástica, evidenciando el aumento de su resistencia. Fi- nalmente, en la zona de fluencia se puede observar para los niveles de menor compactación, una energía de fluencia mayor, dado que la muestra resiste menos y es más blanda.

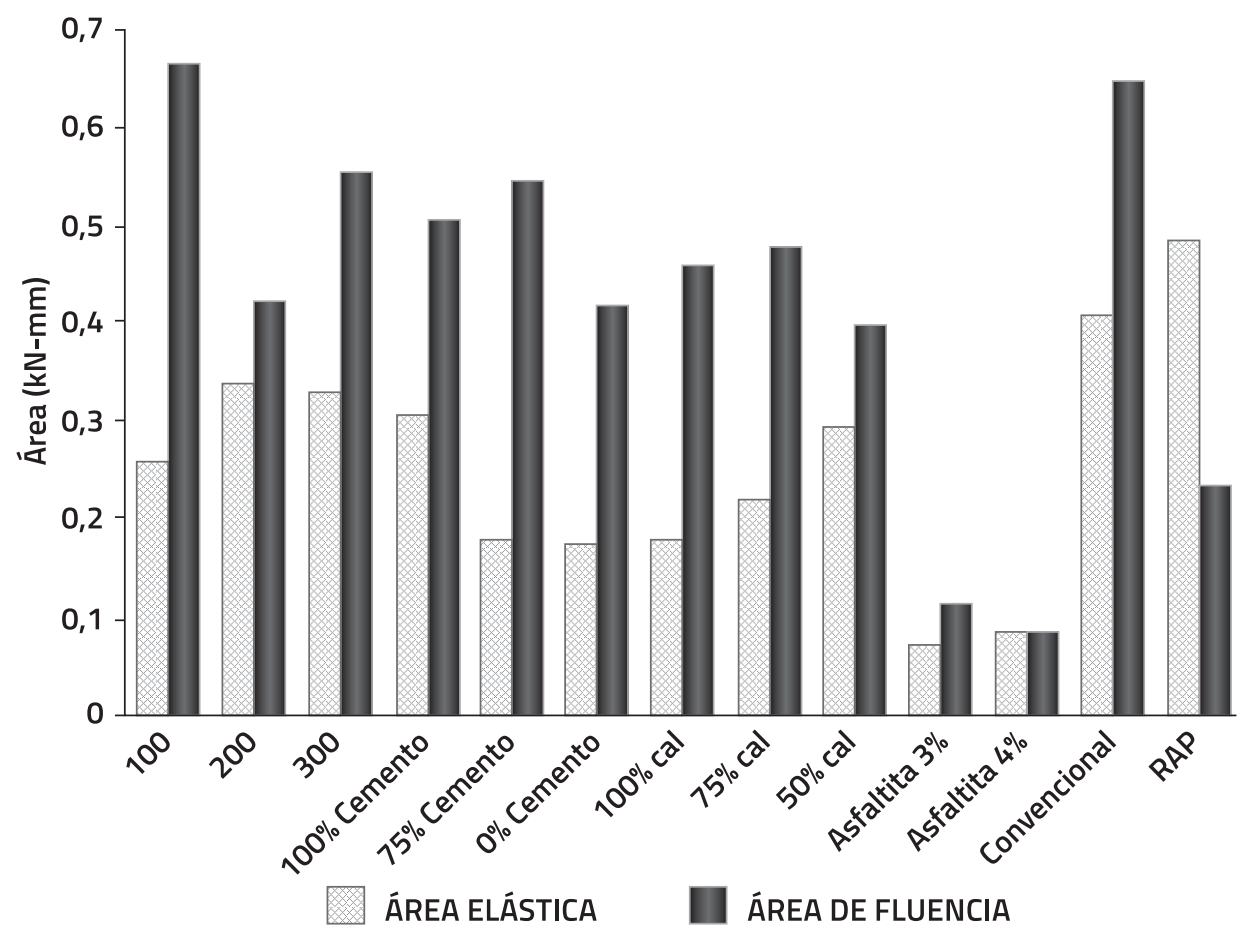

Figura 11. Áreas probetas (Elástica y de fluencia)

Fuente: Original de los autores

En la Figura 12, se observan los resultados del índice de rigidez a tracción (IRT) de las probetas fabricadas con diferentes materiales (Asfaltitas, RAP, convencionales, reemplazo de filler por cemento o cal en diferentes porcentajes y diferentes niveles de compactación). Del parámetro IRT se establece una pseudo-rigidez del material, mostrando qué tan flexible o rígida es la mezcla asfáltica evaluada. En el caso de las muestras estudiadas, se evidencia que los mayores valores corresponden a las mezclas asfálticas fabricadas con RAP y compactadas a 300 giros (Nivel máximo compactado), comportamiento asociado a su material de fabricación y proceso de compactación. Así mismo, se observa en el caso de los 
reemplazos con cemento cambios muy leves en el índice, característica de las mezclas asfálticas rígidas. De otra parte, las muestras fabricadas con asfaltitas presentan los menores valores del parámetro (IRT), mostrando un compor- tamiento característico de ductilidad en las mezclas. Finalmente, este parámetro evidencia el comportamiento de las mezclas asfálticas, haciendo que el ensayo Fénix sea una herramienta útil en la caracterización de las mezclas asfálticas.

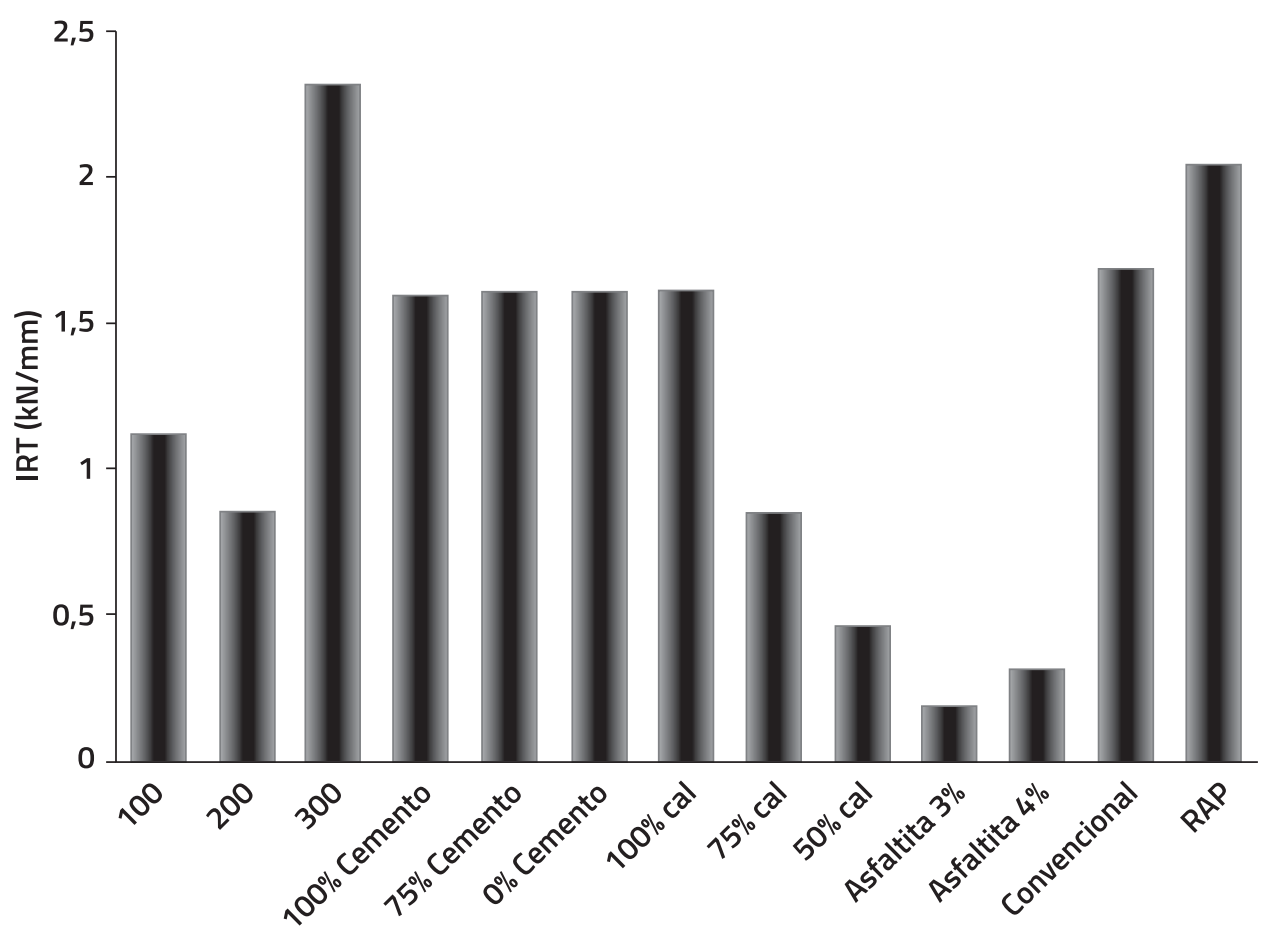

Figura 12. Índice de rigidez a tracción.

Fuente: Original de los autores.

En la Figura 13, se muestran los resultados obtenidos del estudio del índice de energía (IE) de las probetas fabricadas con diferentes materiales (Asfaltitas, RAP, convencionales, reemplazo de filler mineral por cemento o cal en diferentes porcentajes y diferentes niveles de compactación) y las curvas carga-desplazamiento. Del paráme- tro de IE se establece que a mayor índice, la muestra presenta mayor disipación de energía en la zona dúctil. Se puede observar que en la medida que aumenta el nivel de compactación de las mezclas asfálticas (100, 200, 300 giros), el índice disminuye proporcionalmente, indicando que la mezcla se vuelve menos dúctil en función 
del número de giros. De otra parte, en las mezclas asfálticas con reemplazo de filler mineral por cal o cemento se evidencia que a mayor porcentaje de reemplazo, menor es el índice, característica debida a que la muestra se rigidiza. Finalmente, para las asfaltitas el índice es bajo, mostrando que la energía disipada es menor. Nuevamente, se evidencia que el ensayo Fénix y sus características son buenos indicadores del comportamiento a fatiga de las mezclas asfálticas.

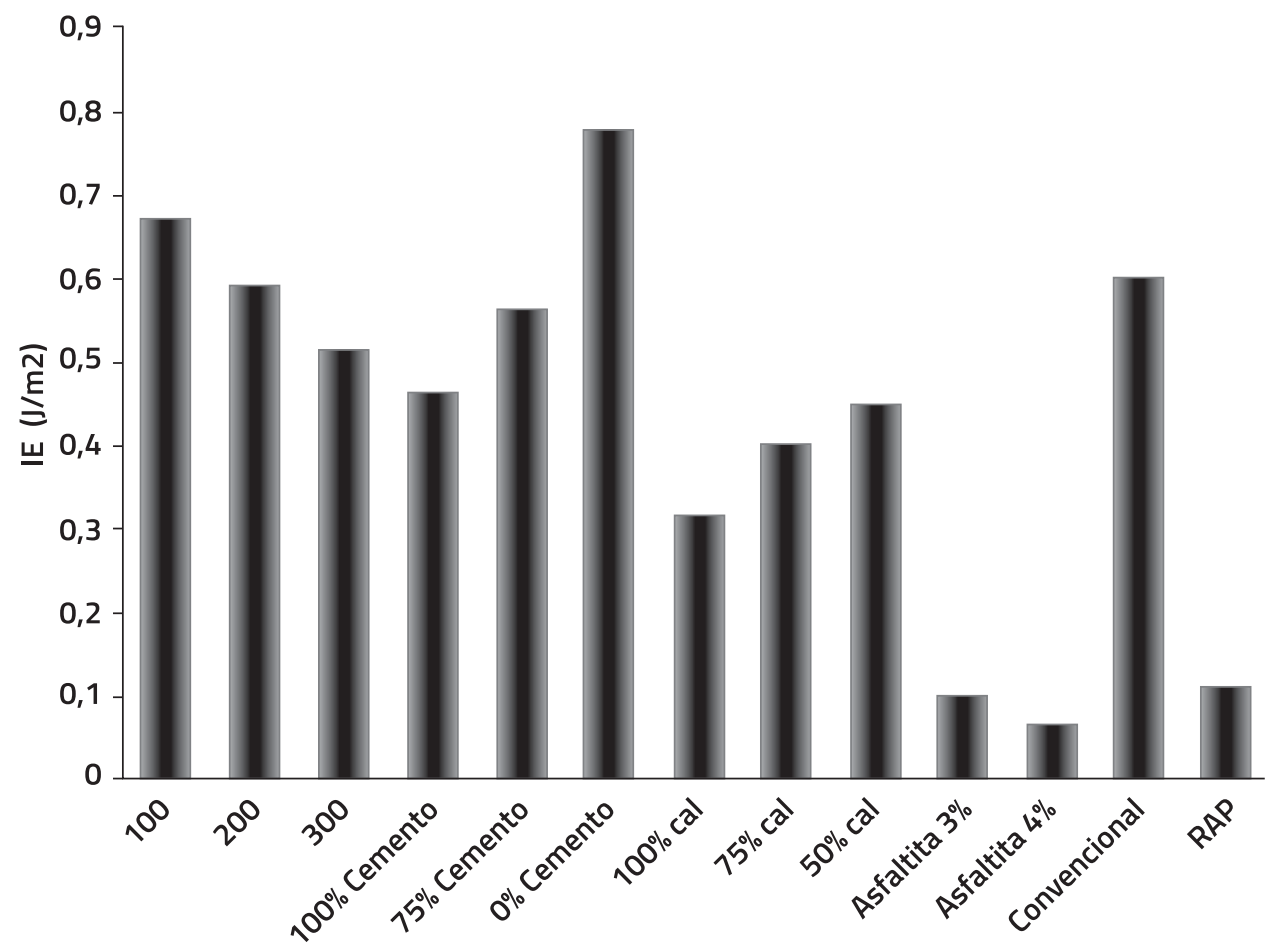

Figura 13. Índice de energía.

Fuente: Original de los autores

\section{CONCLUSIONES}

Con base en el estudio y análisis de las curvas carga-desplazamiento y en los parámetros obtenidos de los ensayos Fénix realizados a las diferentes muestras asfálticas fabricadas, a $15^{\circ} \mathrm{C}$, velocidad controlada de $1 \mathrm{~mm} / \mathrm{min}$, se pudo establecer que:
El procedimiento de ejecución del ensayo Fénix es fácil, sencillo, rápido y económico de realizar.

Los parámetros calculados de las curvas carga-desplazamiento del ensayo Fénix, son sencillos de calcular e indican el comportamiento de las mezclas asfálticas, irre- 
levantemente del material utilizado en su fabricación (Agregados, asfaltos, asfaltita, pavimento asfalto reciclado (RAP), cal, cemento), así mismo del nivel de compactación.

El ensayo Fénix es sensible al comportamiento de materiales frágiles dúctiles, permitiendo diferenciar claramente a partir de sus parámetros y curva carga-desplazamiento, leves cambios en la rigidez o ductilidad de las mezclas asfálticas estudiadas.

Los comportamientos mecánicos obtenidos de las mezclas asfálticas con reemplazo de filler por cemento o cal a diferentes porcentajes, son viables de establecer a partir del análisis de los parámetros del ensayo Fénix, más aún, es viable evidenciar el grado de ductilidad con los índices de energía y los índices de rigidez a tracción.

Finalmente, en las probetas compactadas a diferentes niveles de energía, los parámetros del ensayo Fénix evidenciaron los cambios en sus propiedades.

Por último, a partir de los estudios realizados, se puede establecer que dados los resultados de la investigación el ensayo Fénix para caracterizar mezclas asfálticas fabricadas a partir de diferentes materiales, diferentes reemplazos de filler por cemento o cal, diferentes niveles de compactación. Cabe resaltar que aún falta por establecer correlaciones con los ensayos de módulo resiliente (MR), ahuellamiento, fatiga, entre otros.

\section{AGRADECIMIENTOS}

Los autores agradecen a la Universidad Militar Nueva Granada, en especial al Proyecto de Investigación ING-965 y al Grupo de Geotecnia por la ayuda brindada.

\section{REFERENCIAS}

[1] Hoboken, N.J; John Wiley. (2008). Papagiannakis a. T. Pavement design and materials. Vol 1.

[2] Óscar Javier Reyes Ortiz, (2009). Cambios dinámicos y mecánicos de una mezcla asfáltica densa por las propiedades del asfalto y la energía de compactación. Revista Ingeniería y Desarrollo, Número 26.

[3] R. Miro; F. Pérez Jiménez; M. Olivier y F. Moutier. (2002) "Evaluación de la resistencia a las deformaciones plásticas de mezclas formuladas a partir de la normativa española y francesa "Asociación Española de la Carretera, $\mathrm{p} 7$.

[4] Adrián Zacarías García. (2008)." Estudio de las deformaciones permanentes de mezclas asfálticas elaboradas con asfaltos ac-20 y adicionados con polímeros y aditivos, utilizando la pista de ensayo de laboratorio UMICH." Proyecto de grado, (Ingeniero Civil), Facultad de Ingeniería, Universidad Michoacana de San Nicolás de Hidalgo, México.

[5] Asociación Española de Normalización y Certificación, (2007) Normas UNE-EN. 
Áridos para mezclas bituminosas y tratamientos superficiales de carreteras. (AENOR).

[6] Gonzalo Valdés; F. Pérez-Jiménez y R. Botella. (2009). "Ensayo Fénix, una nueva Metodología para medir la resistencia a la fisuración en mezclas asfálticas". Revista de la Construcción, vol. 8, núm. 1, p 114-125 Pontificia Universidad Católica de Chile.

[7] G. Valdés; F. Pérez; R. Miró; A. Martínez y J. Amorós, (2009). Desarrollo de un nuevo ensayo experimental para la evaluación de la resistencia a tracción directa y la energía disipada en el proceso de fractura en mezclas asfálticas". XV Congreso Ibero- Latino Americano del Asfalto. Lisboa 23-27.

[8] Instituto Nacional de Vías. (2002). Especificaciones generales de construcción para carreteras. Bogotá: Editorial, Escuela Colombiana de Ingeniería. (INVIAS).

[9] Instituto de Desarrollo Urbano. (2005). Especificaciones técnicas generales de materiales y construcción para proyectos de infraestructura vial y de espacio público de Bogotá. Bogotá. (IDU).

[10] Carolina Rodríguez. (2011) "Incidencia del contenido de vacío de una mezcla asfáltica en sus propiedades mecánicas y dinámicas". Proyecto de grado, (Ingeniero Civil), Facultad de Ingeniería, Universidad Militar Nueva Granada, Colombia.

[11] Jhon Edison Castillo. Sandoval; Carlos Alberto Martínez Alarcón. (2011) "Factibili- dad del empleo de asfaltita proveniente del Departamento de Boyacá en la fabricación de mezclas asfálticas", Proyecto de grado, (Ingeniero Civil), Facultad de Ingeniería, Universidad Militar Nueva Granada, Colombia.

[12] Jeame Liliana Ostos Ascencio; Juan Mauricio Duarte Sanmiguel y Óscar Javier Reyes-Ortiz, (2011) "Comportamiento de mezclas asfálticas de Instituto de Desarrollo Urbano con adición de pavimento reciclado. (RAP)" SCIVI Jornadas de Pavimentos y Mantenimiento Vial Bogotá D.C. Colombia.

[13] Mónica Andrea Bautista Sánchez. (2012)," Efecto de las características del asfalto en el comportamiento de las mezclas asfálticas mdc-2." Proyecto de grado, (Ingeniero Civil), Facultad de Ingeniería, Universidad Militar Nueva Granada, Colombia.

[14] Eliecer Suárez Sánchez. (2012) “Influencia de la relación Filler-Asfalto de mezclado asfaltico". Proyecto de grado, (Ingeniero Civil), Facultad de Ingeniería, Universidad Militar Nueva Granada, Colombia.

[15] G. Valdés; Ramón Botella; Félix E.; Pérez Jiménez;; Adriana, Martínez; Rodrigo Miró, (2011) "Experimental study of recycled asphalt mixtures with high percentages of reclaimed asphalt pavement (RAP)". Journal Construction and Building Materials. Vol 25, pp. 1289-1297.

[16] Gonzalo Valdés, "Evaluación del proceso de fisuración mezclas bituminosas mediante el desarrollo de un nuevo ensayo 
experimental Ensayo Fénix" (2009). Tesis Doctoral. (Ph. D) Universidad de Cataluña, España.

[17] Óscar Javier Reyes Ortiz; Félix E. Pérez Jiménez; Rodrigo Miró Recacens; José Amoros Parras y Santiago Gil Redondo. (2009) "El proyecto Fénix en la UPC. Mezclas semicalientes "XV Congreso Ibero Latino Americano del Asfalto. Lisboa 2327 Noviembre.
[15] Rodrigo Miró; G. Valdés; Adriana, Martínez; Pilar Segura; Celestino Rodríguez. "Evaluation of high modulus mixture behaviour with high reclaimed asphalt pavement (RAP) percentages for sustainable road construction". Journal Construction and Building Materials. Vol 25, pp. 3854-3862. 